\title{
A Broader View of Relativity
}

General Implications of Lorentz and Poincaré Invariance

2nd Edition 


\section{ADVANCED SERIES ON THEORETICAL PHYSICAL SCIENCE A Collaboration between World Scientific and Institute of Theoretical Physics}

Series Editors: Dai Yuan-Ben, Hao Bai-Lin, Su Zhao-Bin

(Institute of Theoretical Physics Academia Sinica)

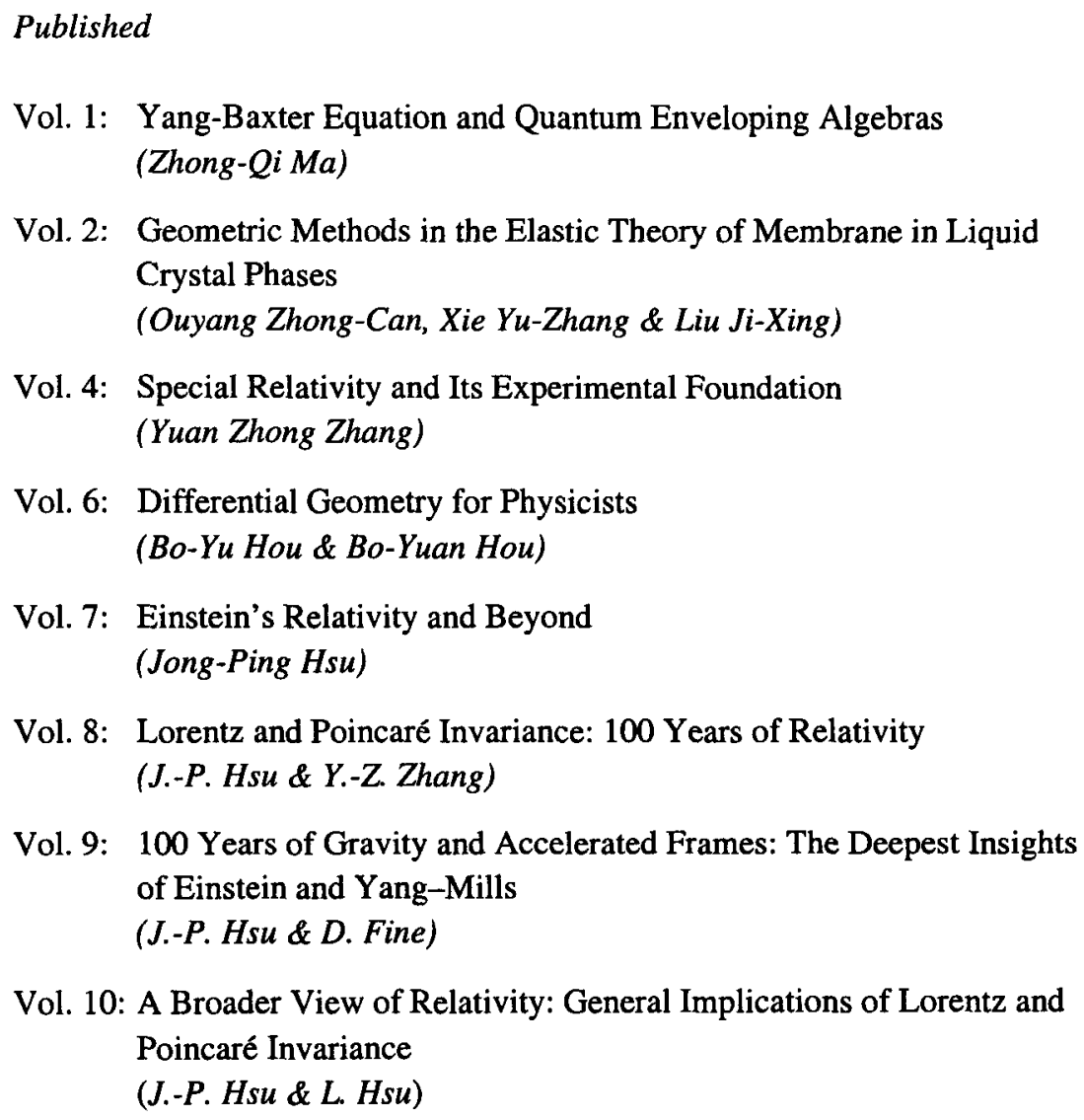

Vol. 9: 100 Years of Gravity and Accelerated Frames: The Deepest Insights of Einstein and Yang-Mills (J.-P. Hsu \& D. Fine)

Vol. 10: A Broader View of Relativity: General Implications of Lorentz and Poincaré Invariance (J.-P. Hsu \& L. Hsu) 


\section{A Broader View of Relativity}

General Implications of Lorentz and Poincaré Invariance

\section{2nd Edition}

Jong-Ping Hsu

University of Massachusetts Dartmouth, USA

\section{Leonardo Hsu}

\section{University of Minnesota, USA}




\section{Published by}

World Scientific Publishing Co. Pte. Ltd.

5 Toh Tuck Link, Singapore 596224

USA office: 27 Warren Street, Suite 401-402, Hackensack, NJ 07601

UK office: 57 Shelton Street, Covent Garden, London WC2H 9HE

\section{Library of Congress Cataloging-in-Publication Data}

A broader view of relativity : general implications of Lorentz and Poincaré invariance / Jong-Ping Hsu, Leonardo Hsu.

p. $\mathrm{cm}$. (Advanced series on theoretical physical science ; v. 10) Includes bibliographical references and index.

ISBN 981-256-651-1 (alk. paper)

1. Special relativity (Physics)--History. 2. Relativity (Physics)--History.

I. Hsu, Leonardo.

QC173.65.H88 2006

$530.11--d c 22$

2006047586

\section{British Library Cataloguing-in-Publication Data}

A catalogue record for this book is available from the British Library.

Copyright $@ 2006$ by World Scientific Publishing Co. Pte. Ltd.

All rights reserved. This book, or parts thereof, may not be reproduced in any form or by any means, electronic or mechanical, including photocopying, recording or any information storage and retrieval system now known or to be invented, without written permission from the Publisher.

For photocopying of material in this volume, please pay a copying fee through the Copyright Clearance Center, Inc., 222 Rosewood Drive, Danvers, MA 01923, USA. In this case permission to photocopy is not required from the publisher. 


\section{A Broader View}

A Broader View of Relativity

A Broader View of Relativity

A Broader View of Relativity

A Broader View of Relativity

A Broader View of Relativity

To

Bonnie Mei-Chu and Leslie

A Broader View of Relativity

A Broader View of Relativity

A Broader View of Relativity

A Broader View of Relativity

A Broader View of Relativity

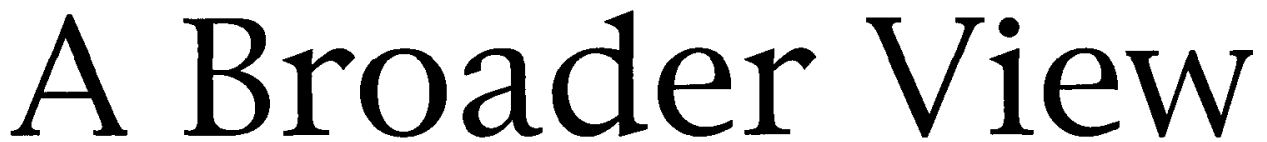


This page is intentionally left blank

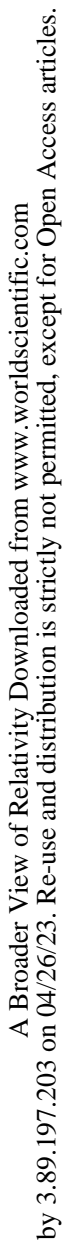


"Those who from time to time send to Nature, as manuscripts intended for publication, proofs that the special theory of relativity must be wrong will in future, at least in the first instance, be referred to the issue of $I$ Nuovo Cimento (74B, 67; 1983) in which Dr. J. P. Hsu from the National Aeronautics and Space Administration's Goddard Space Flight Center* works out some of the consequences of rejecting the notion that the velocity of light is the same in all frames of reference moving relative to each other (or, more strictly, in all inertial frames). For, although Dr. Hsu's professed object is to demonstrate that some "fundamental" constants are more fundamental than others, he embarks on his argument by throwing away the assumption that the velocity of light is constant (and isotropic) for the sake of a system for measuring time which satisfies one of the rudimentary goals of the antirelativists---doing away with the problem of simultaneity. .....

Hsu's starting point, explicitly and emphatically not that of an antirelativist, is that it should be philosophically permissible to require a system for the measurement of what he calls "common time" that should be valid in all relatively moving frames of reference. The procedure is straightforward, and follows Einstein's original discussion of the problem. ..."

NEWS AND VIEWS

Nature Editorial

*It should read "Marshall Space Flight Center" $\quad 303,129$ (1983).

"We are not saying that special relativity is wrong in some way. Instead, taiji relativity shows that relativistic time, or any particular time system for that matter, is not a necessary ingredient of a theory for it to correctly reproduce all known experimental results. The four-dimensional symmetry (i.e., Lorentz and Poincaré invariance) of the physical framework is all that matter."

\section{J. P. Hsu and Leonardo Hsu}

Physics Letters A, 196, 1 (1994), p. 3 
This page is intentionally left blank

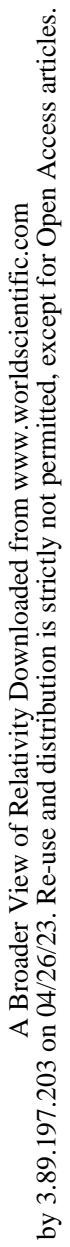




\section{Preface}

In this book, we present a broader view of relativity in order to understand the fundamental concepts of space, time, and truly universal constants based on the smallest number of postulates. Certain new viewpoints, such as the use of a common time for all observers within a four-dimensional symmetry framework, are non-trivial and need time to digest. The impetus for many of the ideas in this book arose from a term paper (see Appendix B) written by LH for a college physics seminar course in the Fall of 1990. The purpose of that paper was to explore the long-standing question of whether the Lorentz transformation could be unambiguously determined from the results of various experiments. The answer turned out to be negative and this result stimulated our collaboration to step back and look at the big picture of relativity and to explore the physical implications of the principle of relativity - a basic theme of this book.

The distinct features of this book are to stress

(A) a broader view of the relationship between the principle of relativity and our concept of time,

(B) a unification of the spacetime transformations for inertial and non-inertial frames based on limiting Lorentz and Poincaré invariance, and

(C) the truly universal and fundamental constants in both inertial and non-inertial frames.

The title of this edition has been changed to "A Broader View of Relativity - General Implications of Lorentz and Poincaré Invariance" to reflect the focus and the main ideas expounded in the book. The revision for this second edition summarizes our collaborative work performed over the past 15 years. In addition to the new chapters $1,7,9,10,20,21,24,25$ and 27, and new appendices A, B and D, many updates and corrections were made. Moreover, the book has heen largely rewritten to reflect updates in our understanding of the conceptual basis of taiji and common relativity including the role that human defined systems of units play in those theories.

Much of the new material is related to the physics of accelerated frames. The physics and spacetime properties of accelerated frames are smoothly connected to those of inertial frames through the limiting Lorentz and Poincaré 
invariance because all accelerated frames become inertial frames in the limit of zero accelerations. Quantum electrodynamics and gravity as a (generalized) Yang-Mills theory are discussed in both inertial and non-inertial frames.

Special acknowledgment is made to Andreas Ernst who helped to correct many typos and translated the original book into German and to Mei-Chu for her tireless support throughout our lives.

This work was supported in part by the Potz Science Fund and the Jing Shin Research Fund of the UMassD Foundation.

Jong-Ping Hsu

(Univ. of Massachusetts Dartmouth)

Leonardo Hsu

(Univ. of Minnesota, Twin Cities) 


\section{Preface to the First Edition}

The purpose of this book is primarily to expound the idea that the first principle of relativity (i.e., the form of a physical law is the same in any inertial frame) taken by itself, implies a new theoretical framework which is consistent with the Lorentz and Poincaré group properties and with all previous experiments. The motivation of this work is not to show that special relativity is wrong in some way, but instead to show that special relativity is in some sense over specified and that removal of the over specification leads to a fresh view of the physical world with new concepts and results which are unobtainable through special relativity.

This new framework uses a four-dimensional symmetry formalism in which the three spatial variables as well as the temporal variable are all expressed in units of length. Furthermore, because it is based on only one postulate, it is the logically simplest theory which has Lorentz and Poincare invariance. This is important because it is absolutely essential to insist that a fundamental physical theory should be derivable from the smallest possible set of basic principles. The 4-dimensional symmetry by itself dictates the kinematics of particles and fields in inertial frames in a manner which can also be extended to non-inertial frames through a limiting procedure.

Three aspects related to this simplest 4-dimensional symmetry framework are discussed:

(A) The first principle of relativity is shown to be the essence of relativity theory. All previous experimental results related to Einstein's theory of special relativity can be derived and understood based solely on this symmetry principle, without invoking an additional postulate regarding the universal constancy of the speed of light. We call such a theory "taiji relativity." As a result, we also find that the number of truly universal and fundamental constants in inertial frames is reduced to two, the atomic fine structure constant $\alpha_{e}=1 / 137.03604$ and a second constant $\mathrm{J}=3.51773 \times 10^{-38} \mathrm{gram} \cdot \mathrm{cm}$. The speed of light (measured in $\mathrm{cm} / \mathrm{sec}$ ) and the Planck constant are found not to be truly fundamental.

(B) The renaissance of the common-sense concept of time $t=t^{\prime}$ is shown to be possible. By embedding this "common time" in a 4-dimensional symmetry framework of the Lorentz and Poincaré groups, rather than the 3-dimensional framework of the Galilean group, we can construct a viable theory ("common 
relativity") which is consistent with all known experiments. Such a theory has certain advantages over special relativity in that we can introduce the notion of the canonical evolution for a system of $\mathrm{N}$ particles and derive the invariant Liouville equation, a basic equation of the statistical physics. This cannot be done, in principle, in special relativity because each particle has its own relativistic time, so that one cannot have a canonical evolution and can \&only derive $\mathrm{N}$ one-particle Liouville equations rather than one single Invariant Liouville equation for $\mathrm{N}$ particles.

$\tilde{g}(C)$ The first principle of relativity can also lead to an 'absolute' theory of ¿spacetime physics in non-inertial frames undergoing a constant linear g gacceleration or a uniform rotation. This is accomplished on the basis of Jimiting 4-dimensional symmetry, the idea that transformations for nonज्ञnertial frames must smoothly reduce to the familiar 4-dimensional transformation of relativity in the limit of zero acceleration. The relativity theory of spacetime for inertial frames is simply the limiting case of such a theory of spacetime ("taiji spacetime") for non-inertial frames. Particle qdynamics and quantization of fields in linearly accelerated frames are fdiscussed. The universal constants in non-inertial frames are found to be the Stame as the ones in inertial frames mentioned above. Thus, $\alpha_{\mathrm{e}}$ and $\mathrm{J}$ are the .

Fruly universal and fundamental constants in the physical world since almost all reference frames in the universe are, strictly speaking, non-inertial.

I want to express my gratitude to Bonnie Hsu for her patience and Fassistance in the preparation of this book. The writing of the book was \%supported in part by the Potz Science Fund and the Jing Shin Research Fund of the University of Massachusetts Dartmouth. I would like to thank the गु Academia Sinica and the Beijing Normal University in Beijing for their Thospitality. I am indebted to Leslie Hsu, George Leung, Wolfhard Kern, John Dowd, Ed King and Kevin Smith for reading many chapters and improving the त़text. I would also like to thank Leonardo $\mathrm{Hsu}$ for his many valuable S.

$\stackrel{\infty}{\infty}$ As the author, I must take sole responsibility for any mistakes in the book and would be grateful to have my attention called to them by readers.

Institute of Theoretical Physics

Academia Sinica, Beijing

November, 1999
J. P. Hsu

(UMassD) 


\section{Overview}

A. The Historical and Physical Context of Relativity Theory

1. Introduction and Overview

2. Space, Time, and Inertial Frames

3. The Nontrivial Pursuit of Earth's Absolute Motion

4. On the Right Track: Voigt, Lorentz, and Larmor

5. The Contributions of Poincaré

6. The Novel Creation of the Young Einstein

B. A Broader View of Relativity: The Central Role of the Principle of Relativity

7. Relativity Based Solely on the Principle of Relativity

8. Common Relativity

9. Experimental Tests I

10. Experimental Tests II

11. Group Properties of Taiji Relativity and Common Relativity

12. Invariant Actions in Relativity Theories and Truly Universal and Fundamental Constants

13. Common Relativity and Many-Body Systems

14. Common Relativity and the $3 \mathrm{~K}$ Cosmic Microwave Background

15. Common Relativity and Quantum Mechanics

16. Common Relativity and Fuzzy Quantum Field Theory

17. Extended Relativity: A Weaker Postulate for the Speed of Light

C. The Role of the Principle of Relativity in the Physics of Accelerated Frames

18. The Principle of Limiting Lorentz and Poincaré Invariance

19. Extended Lorentz Transformations for Frames with Constant-Linear-Accelerations

20. Physical Properties of Spacetime in Accelerated Frames

21. Extended Lorentz Transformations for Accelerated Frames and a Resolution to the "Two-Spaceship Paradox"

22. Dynamics of Classical and Quantum Particles in Constant-Linear-Acceleration Frames

23. Quantization of Scalar, Spinor, and Electromagnetic Fields in Constant-Linear-Acceleration Frames

24. Group and Lie Algebra Properties of Accelerated Spacetime Transformations

25. Coordinate Transformations for Frames with a General-Linear-Acceleration

26. A Taiji Rotational Transformation with Limiting 4-Dimensional Symmetry

27. Epilogue

D. Appendices

A. Systems of Units and the Development of Relativity Theories

B. Can One Derive the Lorentz Transformation From Precision Experiments?

C. Quantum Electrodynamics in Inertial and Non-Inertial Frames

D. Yang-Mills Gravity with Translation Gauge Symmetry in Inertial and Non-inertial Frames 
This page is intentionally left blank

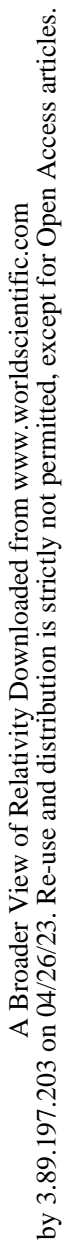




\section{Contents}

$\begin{array}{ll}\text { Preface } & \text { ix }\end{array}$

Preface to the First Edition $\quad$ xi

(A) The Historical and Physical Context of Relativity Theory 1

1. Introduction and Overview 3

1a. Special relativity is NOT incorrect! 3

1b. Idea \#1: Einstein's first postulate of relativity (the principle of relativity) is the only necessary ingredient of a viable theory 4

1c. Idea \#2: The principle of relativity is useful as a limiting principle in the discussion of the physics of accelerated frames

2. Space, Time and Inertial Frames

2a. Space 12

2b. Time 13

2c. Inertial frames of reference $\quad 14$

2d. Coordinate transformations $\quad 15$

2e. Units of space and time 16

3. The Nontrivial Pursuit of Earth's Absolute Motion 19

3a. Newton's frame of absolute rest $\quad 19$

3b. Measuring Earth's velocity 22

4. On the Right Track: Voigt, Lorentz, and Larmor 27

4a. Lorentz's heuristic local time $\quad 27$

4b. Development of the Lorentz transformations 29

5. The Contributions of Poincaré 36

5a. Poincaré's insight into physical time $\quad 36$

5 b. Poincaré and the principle of relativity 38

5c. Poincaré's theory of relativity 41

5d. Conformal transformations and a frame of 'absolute rest' 47

5e. Poincaré's impact on relativity and symmetry principles 51

5f. Retro physics: Past and present views of the ether 54

6. The Novel Creation of the Young Einstein 64

6a. Fresh thoughts from a young mind 64

$6 \mathrm{~b}$. The theory of special relativity 65

6c. Derivation of the Lorentz transformation 66

6d. Relativity of space and time $\quad 68$

6e. The completion of special relativity by Minkowski's idea of
4-dimensional spacetime

$\begin{array}{ll}\text { 6f. Einstein and Poincaré } & 75\end{array}$ 
(B) A Broader View of Relativity: The Central Role of the Principle of Relativity 85

7. Relativity Based Solely on the Principle of Relativity 87

$\begin{array}{ll}\text { 7a. Motivation } & 87\end{array}$

7b. A brief digression: natural units and their physical basis 88

7c. Taiji relativity: A relativity theory based solely on the principle
of relativity

7d. Realization of taiji time $\quad 92$

7e. The conceptual difference between taiji relativity and
special relativity

7f. The role of a second postulate 95

$\begin{array}{lr}\text { 8. Common Relativity } & 100\end{array}$

8a. A new unit for time $\quad 100$

8b. Operationalizing the common-second and the equivalence of
inertial frames

8c. Coordinate transformations in common relativity 104

8d. Physical interpretation of the ligh function b 106

8e. Implications of common time 109

$\begin{array}{lr}\text { 9. Experimental Tests I } & 114\end{array}$

9a. Time intervals versus optical path length $\quad 114$

9b. The Michelson-Morley experiment 114

9c. The Kennedy-Thorndike experiment 118

$\begin{array}{ll}\text { 9d. The Fizeau experiment } & 121\end{array}$

10. Experimental Tests II 128

$\begin{array}{ll}\text { 10a. The Ives-Stilwell experiment } & 128\end{array}$

10b. Atomic energy levels and Doppler shifts in taiji relativity 128

10c. Atomic energy levels and Doppler shifts in common relativity $\quad 130$

10d. Lifetime dilation of cosmic-ray muons 133

10e. The cosmic-ray muon experiment and taiji relativity 134

10f. Decay-length dilation in quantum field theory and taiji relativity

10g. Cosmic-ray muons and common relativity 138

10h. Quantum field theory and the decay length in common relativity $\quad 140$

11. Group Properties of Taiji Relativity and Common Relativity 143

11a. General group properties $\quad 143$

$\begin{array}{ll}11 \mathrm{~b} . \text { Lorentz group properties } & 146\end{array}$

11c. Poincaré group properties $\quad 152$

12. Invariant Actions in Relativity Theories and 158

Truly Fundamental Constants

12a. Invariant actions for classical electrodynamics in relativity theories $\quad 158$

12b. Universal constants and invariant actions 163

12c. Dirac's conjecture regarding the fundamental constants $\quad 165$

12d. Truly fundamental constants $\quad 166$

13. Common Relativity and Many-Body Systems $\quad 170$

13a. Advantages of common time $\quad 170$

13b. Hamiltonian dynamics in common relativity 173 
13c. Invariant kinetic theory of gases

13d. Invariant Liouville equation

13e. Invariant entropy, temperature and the Maxwell-Boltzmann distribution

13f. Invariant Boltzmann-Vlasov equation

13g. Boltzmann's transport equation with 4-dimensional symmetry

13h. Boltzmann's $H$ theorem with 4-dimensional symmetry

14. Common Relativity and the 3K Cosmic Microwave Background

14a. Implications of an invariant and non-invariant Planck's law for blackbody radiation

14b. Invariant partition function

14c. Covariant thermodynamics

14d. The canonical distribution and blackbody radiation

14e. The question of Earth's "absolute" motion relative to the $3 \mathrm{~K}$ cosmic microwave background

15. Common Relativity and Quantum Mechanics

15a. Fuzziness at short distances and the invariant genergy

$15 \mathrm{~b}$. Fuzzy quantum mechanics with an inherent fuzziness in the position of a point particle

15c. Fuzzy point and modified Coulomb potential at short distances

15d. Suppression of the contribution of large momentum states to physical processes

16. Common Relativity and Fuzzy Quantum Field Theory

16a. Fuzzy quantum field theories

16b. Fuzzy quantum electrodynamics based on common relativity

16c. Experimental tests of the 4-dimensional symmetry of special relativity at very high energies

17. Extended Relativity: A Weaker Postulate for the Speed of Light

17a. Four-dimensional symmetry as a guiding principle

17b. Edwards' transformation with Reichenbach's time

17c. Difficulties of Edwards' transformation

17d. Extended relativity: A 4-dimensional theory with Reichenbach's time (a universal 2-way speed of light)

17e. The two basic postulates of extended relativity

17f. Invariant action for a free particle in extended relativity

17g. Comparison of extended relativity and special relativity 256

17h. An unpassable limit and a non-constant speed of light 258

17i. Lorentz group and the space-lightime transformations 259

17j. Decay rate and "lifetime dilation" of unstable particles

(C) The Role of the Principle of Relativity in the Physics of Accelerated Frames

18. The Principle of Limiting Lorentz and Poincaré Invariance

18a. An answer to the young Einstein's question and its implications

18b. Generalizing Lorentz transformations from inertial frames to accelerated frames 
18c. Physical time and 'spacetime clocks' in linearly accelerated frames

18d. Møller's gravitational approach to accelerated transformations

18e. Accelerated transformations with the limiting

Lorentz and Poincaré invariance

19. Extended Lorentz Transformations for Frames with

Constant-Linear-Accelerations

19a. Generalized Møller-Wu-Lee transformation

$19 \mathrm{~b}$. Minimal generalization of the Lorentz transformation: The Wu transformations

19c. A comparison of the generalized MWL and $\mathrm{Wu}$ transformations

19d. Four-momentum and constant-linear-acceleration of an accelerated particle

19e. Experiments on Wu-Doppler effects of waves emitted from accelerated atoms

20. Physical Properties of Spacetime in Accelerated Frames

20a. A general transformation for a CLA frame with an arbitrary $\beta(w)$

20b. The singular wall and horizons in the Wu transformation 300

20c. Generalized Møller-Wu-Lee transformation for an accelerated frame

20d. Decay-length dilations due to particle acceleration

20e. Discussions

21. Extended Lorentz Transformations for Accelerated Frames and a Resolution to the "Two-Spaceship Paradox"

21a. The two-spaceship paradox

21b. Generalized Møller and Wu transformations

21c. Motion and length contraction involving accelerations 324

21d. Discussion

22. Dynamics of Classical and Quantum Particles in

Constant-Linear-Acceleration Frames

22a. Classical electrodynamics in constant-linear-acceleration frames $\quad 330$

22b. Quantum particles and Dirac's equation in a CLA frame 334

22c. Stability of atomic levels against constant accelerations 336

22 d. Electromagnetic fields produced by a charge with a constantlinear-acceleration

22e. Covariant radiative reaction force in special relativity and common relativity

23. Quantizations of Scalar, Spinor, and Electromagnetic Fields

In Constant-Linear-Acceleration Frames

23a. Scalar field in constant-linear-acceleration frames 356

23b. Quantization of scalar fields in CLA frames 359

23c. Quantization of spinor fields in CLA frames 366

23d. Quantization of the electromagnetic field in CLA frames 373

24. Group and Lie Algebra Properties of Accelerated Spacetime Transformations

24a. The Wu transformation with acceleration in an arbitrary direction 
24b. Generators of the Wu transformation in cotangent spacetime

24c. The Wu algebra in a modified momentum space and the classification of particles

25. Coordinate Transformations for Frames with a

General-Linear Acceleration

25a. Spacetime transformations based on limiting Lorentz and Poincaré invariance

25b. Physical implications and discussion

26. A Taiji Rotational Transformation with

Limiting 4-Dimensional Symmetry

402

26a. A smooth connection between rotational and inertial frames

26b. A taiji rotational transformation with limiting 4-dimensional symmetry

26c. Physical properties of the taiji rotational transformation

26d. The metric tensors for the spacetime of rotating frames

26e. The invariant action for electromagnetic fields and charged particles in rotating frames and truly fundamental constants

26f. The 4-momentum and the 'lifetime dilation' of a particle at rest in a rotating frame

27. Epilogue

(D) Appendices

A. Systems of Units and the Development of Relativity Theories

Aa. Units, convenience and physical necessity $\quad 425$

Ab. Time, length and mass $\quad 426$

Ac. Other SI base units $\quad 430$

Ad. Other units $\quad 434$

Ae. Status of the fundamental constants $\quad 434$

Af. Discussion and conclusion $\quad 436$

B. Can one Derive the Lorentz Transformation from
Precision Experiments?

Ba. Introduction $\quad 442$

Bb. Three classical tests of special relativity 443

Bc. Deriving the Lorentz transformation? 446

Bd. A more general form $\quad 455$

Be. Discussions and conclusions 462

C. Quantum Electrodynamics in Both Linearly Accelerated and Inertial Frames

Ca. Quantum electrodynamics based on taiji relativity 465

Cb. Experimental measurements of dilations of decay-lengths and decay-lifetimes in inertial frames

Cc. Quantum electrodynamics of bosons in accelerated and inertial frames 
Cd. Feynman rules for QED with fermions in both CLA and inertial frames

Ce. Some QED results in both CLA and inertial frames

D. Yang-Mills Gravity with Translation Gauge Symmetry in Inertial and Non-inertial Frames

Da. Translation gauge transformations and an 'effective metric tensor' in flat spacetime

$\mathrm{Db}$. Yang-Mills theory with translation gauge symmetry

489

Dc. Gravitational action with quadratic gauge-curvature

490

Dd. Linearized equations of the tensor field and the Hamilton-Jacobi equation for particles

De. The gauge field equation in inertial and non-inertial frames

Df. Perihelion shifts and bending of light

498

Dg. The Yang-Mills gravitational force 